\title{
G- Frame Operator in C* Algebra
}

\author{
P. Kalyani \\ Department of Mathematics, Aurora's Technological and Research Institute, Uppal, Hyderabad
}

\begin{abstract}
The g-frame operator for $g$-frames in $C^{*}$ algebra is introduced. The results on $g$-frame operators are proved. Frame identities are shown. Result on direct sum of $g$-frame operators on direct sum of Hilbert Spaces is presented.

\section{Introduction}

Frames are generalization of bases .D. Han and D. R. Larson have developed a number of basic concepts of operator theoretic approach to frame theory in $C^{*}$ algebra. Peter G Casazza presented a tutorial on frame theory and he suggested the major directions of research in frame theory. Radu V. Balan and Peter G. Casazza have analyzed decomposition of a normalized tight frame and obtained identities for frames. A. Najati and A. Rahimi have developed the generalized frame theory and introduced methods for generating g-frames of a $C^{*}$ algebra.
\end{abstract}

1.1. Banach Algebra:- A Banach Algebra is a complex Banach space A together with an associative and distributive multiplication such that $\lambda(\mathrm{ab})=\lambda(\mathrm{a}) \lambda(\mathrm{b})$,

$\|a b\| \leq\|a\|\|b\| \forall \mathrm{a}, \mathrm{b} \in A$ and $\lambda \in C$

For any $\mathrm{x}, x^{1}, y, y^{1} \in A$ we have $\left\|x y-x^{1} y^{1}\right\| \leq$

$\|x\|\left\|y-y^{1}\right\|+\left\|x-x^{1}\right\|$

The algebra $\mathrm{A}$ is said to be commutative if $\mathrm{ab}=\mathrm{ba} \forall a, b \in A$

1.2. Definition (Involution of an algebra):- Let $A$ be a Banach algebra .An involution on $A$ is a map *: $A \rightarrow A$ such that

1. $a^{*^{*}}=\mathrm{a}$

2. $(\lambda a+\mu b)^{*}=\bar{\lambda} \mathrm{a}^{*}+\bar{\mu} \mathrm{b}^{*}$

3. $(a b)^{*}=\mathrm{b}^{*} \mathrm{a} *$

1.3. Definition:- ( $\mathrm{C}^{*}$ algebra) If $\mathrm{A}$ is a Banach algebra with involution and also $\|a a *\|=\|a\|^{2}$ then $\mathrm{A}$ is called a c* algebra.

Example:- $\mathrm{C}(\mathrm{X})$ let $\mathrm{X}$ be a compact space and $\mathrm{C}(\mathrm{X})$ is a Banach space of all complex valued functions on $\mathrm{X}$ with norm $\|f\|=\sup _{x \in X}|f(x)|$ Multiplication on $\mathrm{C}(\mathrm{X})$ is defined as pointwise ief.g(x)=f(x)g(x)

And involution by complex conjugation $\mathrm{f}^{*}(\mathrm{x})=\overline{f(x)}$

\section{G- Frame And G-Frame Operator}

Throughout this paper $\left\{A_{j} \mathrm{j} \in \mathrm{J}\right\}$ will denote a sequence of $\mathrm{C}^{*}$ algebras .Let $\mathrm{L}\left(\mathrm{A}, A_{j}\right)$ be a collection of bounded linear operators from $\mathrm{A}$ to $A_{j}$ and $\left\{\Delta_{j} \in \mathrm{L}\left(\mathrm{A}, A_{j}\right) ; \mathrm{j} \in \mathrm{J}\right\}$ we obtain some characterization of $\mathrm{g}$-frame operator. They are the generalizations of results of frame operator.

2.1. Definition: - A sequence of operators $\left\{\Delta_{j}\right\}_{j \in J}$ is said to be $\mathrm{g}$-frame for $\mathrm{C}^{*}$ algebra $\mathrm{A}$ with respect to sequence of $\mathrm{C}^{*}$ algebras $\left\{A_{j}, \mathrm{j} \in \mathrm{J}\right\}$ if there exists two constants $0<\mathrm{A} \leq \mathrm{B}<\infty$ for any vector $\mathrm{f} \in \mathrm{H}$,

$$
\mathrm{A}\|\bar{f}\|^{2} \leq \sum_{j \in J}\left\|\Delta_{j} \bar{f}\right\|^{2} \leq \mathrm{B}\left\|f^{2}\right\| \text { where } \bar{f}(\mathrm{x})=\mathrm{f}^{*}(\mathrm{x})
$$

The above inequality is called a g-frame inequality. The numbers A, B are called the lower frame bound and upper frame bound respectively.

2.2. Definition: -A g-frame for $\left\{\Delta_{j}\right\}_{j \in J}$ is said to be g-tight frame if $\mathrm{A}=\mathrm{B}$, then we have

$$
\mathrm{A}\|\bar{f}\|^{2}=\sum_{j \in J}\left\|\Delta_{j} \bar{f}\right\|^{2} \text { for all } \mathrm{f}^{*} \in \mathrm{A}
$$

2.3. Definition: - A g-frame $\left\{\Delta_{j}\right\}_{j \in J}$ for $\mathrm{A}$ is said to be a g-normalized tight frame for $\mathrm{A}$ if $\mathrm{A}=\mathrm{B}=1$. Then we have $\|\bar{f}\|^{2}=\sum_{j \in J}\left\|\Delta_{j} \bar{f}\right\|^{2}$ for all $\mathrm{f} \in \mathrm{H}$

2.4. Definition: -Let $\left\{\Delta_{j}\right\}_{j \in J}$ be a g-frame for $\mathrm{c}^{*}$ algebra. G-frame operator

$$
S^{g}: \mathrm{A} \rightarrow \mathrm{A} \text { is defined as }
$$

$$
S^{g} f=\sum_{j \in J} \Delta_{j}^{*} \Delta_{j} \mathrm{f}^{*} \text { for all } \mathrm{f}^{*} \in \mathrm{A}
$$


By using above definitions we have the following theorems.

\section{Main Result}

3.1. Theorem: If $S^{g}$ is a g-frame operator, then we have

1) $<S^{g} \mathrm{f}, \mathrm{f}>=\sum_{j \in J}\left\|\Delta_{j} f\right\|^{2}$ for all $\mathrm{f} \in \mathrm{A}$

2) $S^{g}$ is a positive operator

3) $S^{g}$ is a self-adjoint operator.

Proof: $-S^{g}$ is a g-frame operator means $S^{g} f=\sum_{j \in J} \Delta_{j}^{*} \Delta_{j}$ f* for all $f^{*} \in \mathrm{A}$

1) $\quad<S^{g} \mathrm{f}, \mathrm{f}>=<\sum_{j \in J} \Delta_{j}^{*} \Delta_{j} \mathrm{f}^{*} \mathrm{f}, \mathrm{f}>$

$=\sum<\Delta_{j}^{\times} \Delta_{j} f, \Delta_{j}^{\times} \Delta_{j} f>$

$=\sum\left\|\Delta_{j} \mathrm{f}\right\|^{2}\left[\because<\mathrm{x}, \mathrm{x}>=\left\|x^{2}\right\|\right]$

(2) Clearly $S^{g}$ is a positive operator by definition

(3) It is left to the reader

3.2. Theorem: Suppose $\left\{\Delta_{j}\right\}_{j \in J}$ is a g- frame if and only if $\mathrm{AI} \leq S^{g} \leq \mathrm{BI}$ and $\left\{\Delta_{j}\right\}_{j \in J}$ is a g-normalized tight frame if and only if $S^{g}=\mathrm{I}$ where I is an identity operator on A.

Proof: Since $\left\{\Delta_{j}\right\}_{j \in J}$ is a g-frame so we have

$$
\begin{gathered}
\mathrm{A}\left\|\bar{f}^{2}\right\| \leq\left\|\Delta_{j} \bar{f}\right\|^{2} \leq \mathrm{B}\left\|\bar{f}^{2}\right\| \text { for all } \bar{f} \in \mathrm{A} \\
\begin{array}{c}
\text { Consider }\langle A I \bar{f}, \bar{f}\rangle=\mathrm{A}\langle\bar{f}, \bar{f}\rangle=\mathrm{A}\left\|\bar{f}^{2}\right\| \leq \sum_{j \in J} \llbracket \Delta_{j} \bar{f} \rrbracket^{2} \leq B\|f\|^{2} \\
=\mathrm{B}\langle\bar{f}, \bar{f}\rangle=\langle B I \bar{f}, \bar{f}\rangle
\end{array}
\end{gathered}
$$

Conversely suppose $\mathrm{AI} \leq S^{g} \leq B I$

$\Rightarrow\langle A I \bar{f}, \bar{f}\rangle \leq\left\langle S^{g} \bar{f}, \bar{f}\right\rangle \leq\langle I B \bar{f}, \bar{f}\rangle$ for all $\bar{f} \in \mathrm{A}$

$\Rightarrow \mathrm{A}\left\|\bar{f}^{2}\right\| \leq \sum_{j \in J}\left\|\Delta_{j} \bar{f}\right\|^{2} \leq B\|\bar{f}\|^{2}$

Which implies $\left\{\Delta_{j}\right\}_{j \in J}$ is a g-frame for A.

Suppose $\left\{\Delta_{j}\right\}_{j \in J}$ is a g-normalized tight frame for A

$\Leftrightarrow \sum_{j \in J}\left\|\Delta_{j} \bar{f}\right\|^{2}=\|\bar{f}\|^{2}$ for all $\bar{f} \in \mathrm{A}$

If and only if $\left\langle s^{g} f, f\right\rangle=\langle I f, f\rangle$

If and only if $S^{g}=\mathrm{I}$

Note: We can easily see that frame operator $S^{g}$ is invertible and $S^{g^{-1}}$ is a positive operator.

3.3. Theorem: Let $S^{g}$ be a g-frame operator for the g-frame $\left\{\Delta_{j}\right\}_{j \in J}$ with frame bounds A,B in the

$$
\mathrm{C}^{*} \text { algebra A. Then } B^{-1} \mathrm{I} \leq S^{g^{-1}} \leq A^{-1} \mathrm{I}
$$

Proof: Since $\left\{\Delta_{j}\right\}_{j \in J}$ is a g-frame for $\mathrm{C}^{*}$ algebra $\mathrm{A}$, we have $\mathrm{AI} \leq S^{g} \leq \mathrm{BI}$

Since $\mathrm{AI} \leq S^{g} \Rightarrow 0 \leq\left(S^{g}-\mathrm{AI}\right) S^{g^{-1}} \Rightarrow 0 \leq \mathrm{I}-\mathrm{A} S^{g^{-1}}$

$$
\Rightarrow S^{g^{-1}} \leq A^{-1} \mathrm{I}
$$

Similarly, we can prove $B^{-1} \mathrm{I} \leq S^{g^{-1}}$

Hence from (1) and (2) $B^{-1} \mathrm{I} \leq S^{g^{-1}} \leq A^{-1} \mathrm{I}$

3.4. Theorem: A sequence of operators $\left\{\Delta_{j}\right\}_{j \in J}$ where $\overline{\Delta_{j}}=\Delta_{j} S^{g^{-1}}$ is a G-frame for C* algebra with frame bounds $1 / \mathrm{B}$ and $1 / \mathrm{A}$

Proof: Consider $\sum_{j \in J}\left\|\Delta_{j}^{*} f\right\|^{2}=\sum_{j \in J}\left\|\bar{\Delta}_{j} f\right\|^{2}$

$$
\begin{aligned}
& =\sum_{j \in J}\left\|\Delta_{j} S^{g^{-1}} f\right\|^{2} \\
& =\sum_{j \in J}\left\langle\Delta_{j}^{*} \Delta_{j} S^{g^{-1}} f, S^{g^{-1}} f\right\rangle \\
& =\left\langle\sum_{j \in J} \Delta_{j}{ }^{*} \Delta_{j} S^{g^{-1}} f, S^{g^{-1}} f\right\rangle \\
& =\left\langle S^{g} S^{\left.g^{-1} f, S^{g^{-1}} f\right\rangle}\right. \\
& =\left\langle f, S^{g^{-1}} f\right\rangle \leq \frac{1}{A}\|f\|^{2} \\
& \Rightarrow \sum_{j \in J}\left\|\bar{\Delta}_{j} f\right\|^{2} \leq \frac{1}{A}\|f\|^{2} \\
& \|f\|^{2}=\left\langle\sum_{j \in J} \bar{\Delta}_{j}{ }^{*} \Delta_{j} f, f\right\rangle=\sum_{j \in J}\left\langle\Delta_{j}{ }^{*} \Delta_{j} f, f\right\rangle
\end{aligned}
$$




$$
\begin{gathered}
=\sum_{j \in J}\left\langle\Delta_{j} f \bar{\Delta}_{j} f\right\rangle \\
\leq\left[\sum_{j \in J}\left\|\Delta_{j} f\right\|^{2}\right]^{\frac{1}{2}}\left[\sum_{j \in J}\left\|\bar{\Delta}_{j} f\right\|^{2}\right]^{\frac{1}{2}} \\
\leq \sqrt{B}\|f\|\left[\sum_{j \in J}\left\|\bar{\Delta}_{j} f\right\|^{2}\right]^{1 / 2} \\
\Rightarrow\|f\|^{2} \leq \sqrt{B}\|f\|\left[\sum_{j \in J}\left\|\bar{\Delta}_{j} f\right\|^{2}\right]^{1 / 2} \\
\Rightarrow \frac{1}{B}\|f\|^{2} \leq \sum_{j \in J}\left\|\bar{\Delta}_{j} f\right\|^{2}
\end{gathered}
$$

Hence $\Rightarrow \frac{1}{B}\|f\|^{2} \leq \sum_{j \in J}\left\|\bar{\Delta}_{j} f\right\|^{2} \leq \frac{1}{A}\|f\|^{2}$

Which shows that the sequence of operators $\left\{\bar{\Delta}_{j}\right\}_{j \in J}$ is a g-frame for the $\mathrm{C}^{*}$ algebra A with frame bounds $\frac{1}{B}$ and $\frac{1}{A}$

3.5. Theorem: - Let $\left\{\Delta_{j}\right\}_{j \in J}$ be a $\mathrm{g}$-frame for $\mathrm{C}^{*}$ algebra $\mathrm{A}$ with respect to $\left\{A_{j}, \mathrm{j} \in \mathrm{J}\right\}$ and $\mathrm{V} \in \mathrm{B}(\mathrm{H})$ be an invertible operator. Then $\left\{\Delta_{j} \mathrm{~V}\right\}_{j \in J}$ is a $\mathrm{G}$-frame for $\mathrm{A}$ with respect to $\left\{A_{j}, \mathrm{j} \in \mathrm{J}\right\}$ and its $\mathrm{g}$-frame operator is $V^{*} S^{g} \mathrm{~V}$.

Proof: - Since $\mathrm{V} \in \mathrm{B}(\mathrm{H}), \forall \mathrm{f} \in \mathrm{H}$, we have $V f \in \mathrm{H}$ given that $\left\{\Delta_{j}\right\}_{j \in J}$ is a $\mathrm{G}$-frame for $\mathrm{H}$, so for all $V \mathrm{f} \in \mathrm{H}$ we have $\|\mathrm{Vf}\|^{2} \leq \sum_{j \in J}\left\|\Delta_{j} V f\right\|^{2} \leq \mathrm{B}\|\mathrm{Vf}\|^{2}$

Since $\mathrm{V}$ is invertible operator, therefore we have

$$
\|\mathrm{Vf}\|^{2} \leq\|\mathrm{V}\|^{2}\|\mathrm{f}\|^{2} \text { and }\left\|V^{-1}\right\|^{-2}\|\mathrm{f}\|^{2} \leq\|\mathrm{Vf}\|^{2}
$$

By above inequalities, the equation become

$$
\mathrm{A}\left\|V^{-1}\right\|^{-2}\|\mathrm{f}\|^{2} \leq \sum_{j \in J}\left\|\Delta_{j} V f\right\|^{2} \leq \mathrm{B}\|\mathrm{V}\|^{2}\|\mathrm{f}\|^{2}, \forall \mathrm{f} \in \mathrm{H}
$$

$$
\Rightarrow\left\{\Delta_{j} V\right\}_{j \in J} \text { is a } \mathrm{g} \text {-frame for } \mathrm{A} \text {. }
$$

For each, $f \in A$. We have $S^{g} \mathrm{~V} f=\sum_{j \in J} \Delta_{j}^{*} \Delta_{j} \mathrm{Vf}$

$$
\Longrightarrow V^{*} S^{g} \mathrm{Vf}=\sum_{j \in J} V^{*} \Delta_{j}^{*} \Delta_{j} \mathrm{Vf}
$$

$\Rightarrow V^{*} S^{g} \mathrm{~V}$ is a g-frame operator for the frame $\left\{\Delta_{j} V\right\}_{j \in J}$

Frame Identities for $\mathrm{g}$-frames

3.6. Proposition: If, $T_{1}$ and $T_{2}$ are two operators in a $\mathrm{C}^{*}$ algebra A satisfying $T_{1}+T_{2}=\mathrm{I}$,

$$
\text { Then } T_{1}-T_{2}=T_{1}^{2}-T_{2}^{2}
$$

Proof: Consider $T_{1}-T_{2}=T_{1}-\left(\mathrm{I}-T_{1}\right)=T_{1}^{2}-\left(\mathrm{I}-2 T_{1}+T_{1}^{2}\right)$

$$
\begin{aligned}
& =T_{1}^{2}-\left(\mathrm{I}-T_{1}\right)^{2} \\
& =T_{1}^{2}-T_{2}^{2}
\end{aligned}
$$

3.7. Theorem: - Let $\left\{\Delta_{j}\right\}_{j \in J}$ be a g-normalize d tight frame for A for ICJ, then

$$
\sum_{j \in J}\left\|\Delta_{j} f\right\|^{2}=\left\|\sum_{j \in J} \Delta_{j}^{*} \Delta_{j}\right\|^{2} \Leftrightarrow S_{I}^{g} S_{I^{c}}^{g}=0
$$

Proof: Consider $\sum_{j \in J}\left\|\Delta_{j} f\right\|^{2}=\left\|\sum_{j \in J} \Delta_{j}^{*} \Delta_{j}\right\|^{2} \Leftrightarrow \sum_{j \in J}\left\|\Delta_{j} \mathrm{f}\right\|^{2}$

$$
\Leftrightarrow\left\|\sum_{j \in J} \Delta_{j}^{*} \Delta_{j}\right\|^{2}=0
$$

$$
\Leftrightarrow \sum_{j \in J}\left\|\Delta_{j} \mathrm{f}\right\|^{2}-<\sum_{j \in J} \Delta_{j}^{*} \Delta_{j} f, \sum_{j \in J} \Delta_{j}^{*} \Delta_{j} f>=0
$$

$\Leftrightarrow<\mathrm{S}_{\mathrm{I}}^{\mathrm{g}} \mathrm{f}, \mathrm{f}>-<\mathrm{S}_{\mathrm{I}}^{\mathrm{g}} \mathrm{f}, \mathrm{S}_{\mathrm{I}}^{\mathrm{g}} \mathrm{f}>=0$

$\Leftrightarrow<\left(S_{I}^{\mathrm{g}}-\mathrm{S}_{\mathrm{I}}^{\mathrm{g}}\right)^{2} \mathrm{f}, \mathrm{f}>=0$

$\Leftrightarrow S_{I}^{\mathrm{g}}\left(\mathrm{I}-\mathrm{S}_{\mathrm{I}}^{\mathrm{g}}\right) \mathrm{f}=0$

$\Leftrightarrow S_{I}^{g} S_{I^{c}}^{g}=0$ for all $\mathrm{f} \in \mathrm{H}$

3.8. Theorem: Let $\left\{\Delta_{\mathrm{j}}\right\}_{\mathrm{j} \in \mathrm{J}}$ be a g-normalized tight frame for $\mathrm{H}$, for ICJ with respect to $\left[H_{j}, \mathrm{j} \in \mathrm{J}\right]$. Then for ICJ and for all $\mathrm{f} \in \mathrm{H}$.

$$
\sum_{j \in J}\left\|\Delta_{j} \mathrm{f}\right\|^{2}+\left\|S_{I^{c}}^{g} \mathrm{f}\right\|^{2}=\sum_{j \in J}\left\|\Delta_{j} \mathrm{f}\right\|^{2}+\left\|S_{I}^{g} \mathrm{f}\right\|^{2}
$$

Proof: Science $\left\{\Delta_{\mathrm{j}}\right\}_{\mathrm{j} \in \mathrm{J}}$ is a g-normalized tight frame for $\mathrm{H}$,

Therefore $S^{g}=\mathrm{I}$ and $S_{I}^{g}+S_{I^{c}}^{g}=\mathrm{I}$, for all $\mathrm{f} \in \mathrm{H}$. 
Consider $\sum_{j \in J}\left\|\Delta_{j} \mathrm{f}\right\|^{2}+\left\|S_{I^{c}}^{g} \mathrm{f}\right\|^{2}=\left\langle S_{I}^{g} \mathrm{f}, \mathrm{f}\right\rangle+\left\langle S_{I^{c}}^{g} \mathrm{f}, S_{I^{c}}^{g} \mathrm{f}\right\rangle$

$$
\begin{aligned}
& =<\left(S_{I}^{g}+S_{I^{c}}^{g}\right)^{2} \mathrm{f}, \mathrm{f}> \\
& =<\left(S_{I}^{g}+\left(\mathrm{I}-S_{I}^{g}\right)^{2} \mathrm{f}, \mathrm{f}>\right. \\
& \left.=<\left(\mathrm{I}-S_{I}^{g}+S_{I}^{g}\right)^{2} \mathrm{f}, \mathrm{f}\right\rangle \\
& =<\left(S_{I^{c}}^{g}+S_{I}^{g}\right)^{2} \mathrm{f}, \mathrm{f}> \\
& =<S_{I^{c}}^{g} \mathrm{f}, \mathrm{f}>+<S_{I}^{g} \mathrm{f}, S_{I}^{g} \mathrm{f}> \\
& =\sum_{j \in I^{c}}\left\|\Delta_{j} \mathrm{f}\right\|^{2}+\left\|S_{I}^{g} \mathrm{f}\right\|^{2}
\end{aligned}
$$

\section{References}

[1]. M.R.Abdollahpour ,M.H. Faroughi and A.Rahimi “ PG-Frames in Banach spaces” Methods of Functional analysis and topology

[2]. Vol.13 no.3(2007),pp.201-210

[3]. R.Balan P.G.Casazza, D.Edidin and G.Kutynoik “ Decomopostion of frames and a new frame identity “ Wavelet XI (San

[4]. Diego),CA(2005) pp.379-388 SPIE Proc 5914 ,SPIE Bellinginam WA.

[5]. P.G.Casazza, "The art of Frame theory" ,Taiwanese Journal of Mathematics Vol.4,No.2(2000) pp 129-201

[6]. D.Han and D.R.Larson "Frames Bases and Group Representations", Memories Ams Nov 7 (2000) Providence RI

[7]. A.Najati and A.Rahimi "Generalized frames in Hilbert spaces” ,Bulletin of the Iranian Mathematical society Vol.35,No.1(2009) pp.97- 109 .

[8]. A.Najati and M.H.Faroughi “ P- frames of subspaces of separable Hilbert spaces” ,South east Asian Bulletin of Mathematics 31(2007) pp.713-726.

[9]. G. Upender Reddy and N.Gopal Reddy, "Some results of Frame operator in Hilbert space", Journal of Mathematical Education, Volume XLV, No..3 September 2011. 